$$
\begin{gathered}
\text { N-45-C2 } \\
\text { NAG } 5-1699 \\
83748 \\
P-19
\end{gathered}
$$

\title{
SATELLITE OBSERVATION AND MAPPING OF WINTERTIME OZONE VARIABILITY IN THE LOWER STRATOSPHERE
}

\section{by}

Marvin A. Geller and Yuechen Chi

Institute for Terrestrial and Planetary Atmospheres

State University of New York at Stony Brook Stony Brook, New York

Richard B. Rood, Anne R. Douglass, and Jack A. Kaye Atmospheric Chemistry and Dynamics Branch

NASA Goddard Space Flight Center

Greenbelt, Maryland

Dale J. Allen

Applied Research Corporation

Landover, Maryland 


\begin{abstract}
Comparison is made between 30 mbar ozone fields that are generated by a transport-chemistry model utilizing the winds from the Goddard Space Flight Center stratospheric data assimilation system (STRATAN), observations from the LIMS instrument on Nimbus 7 , and the ozone fields that result from "flying" a mathematical simulation of LIMS observations through the transport-chemistry model ozone fields. The modeled ozone fields are found to resemble the LIMS observations, but the model fields show much more temporal and spatial structure than do the LIMS observations. The "satellite-mapped" model results resemble the LIMS observations much more closely. These results are very consistent with the earlier discussions of satellite space-time sampling by Salby.
\end{abstract}




\section{Introduction}

Remote sensing of the stratosphere from orbiting satellites has proven to be very valuable in advancing our understanding of stratospheric chemistry and transport processes. Using satellite data is not always so straightforward, however. Orbiting satellites observe the atmosphere in an asynoptic manner; that is to say, they observe different points in space sequentially in time along their orbit. Yet, much of the mathematical framework used in the atmospheric sciences relies on the use of synoptic fields. For instance, the three-dimensional equations of motion and transport are cast in the form of temporal and spatial derivatives of field variables so that all of these field variables must be known as a function of the three spatial variables and time. Thus, one problem that was appreciated very early on in the use of satellite data was the need to generate the best "synoptic maps" from these asynoptic satellite data.

In a series of papers, Salby (1982a, 1982b, 1987, 1989) developed a mathematical basis for calculating the spatial and temporal resolutions that can be obtained from asynoptic satellite data. He also developed a method for optimal (in the sense of least aliasing) transformation of asynoptic observations into "synoptic maps," and examined some of the problems to be encountered in satellite observation and the subsequent mapping of stratospheric satellite data. Many of the results of this paper are implied by the results of Salby.

Our investigation is specifically focussed on how faithfully mapped satellite data represents the actual distribution of stratospheric constituents. To do this, we compare the results of a transport-chemistry model for ozone with satellite observations of ozone. Such a comparison can be carried out since the wind fields that are used in the transport-chemistry model are derived from measurements using an assimilation model and represent the conditions that existed at the actual times of satellite observation. The models used in this paper will be described further in Section 2. A mathematical simulation of asynoptic "satellite observation" of the model results is made using similar parameters to those for the actual satellite observations. This is described in Section 3. The "satellite observations" of the model are then mapped using the same techniques that were used in the mapping of the actual satellite data. These mapping methods are described in Section 4. Results are given 
in Section 5, and Section 6 is a discussion and summary of these results.

\section{The Model}

The models used in this paper are the same as those described by Rood et al. (1991), so our descriptions will be brief, and the reader is referred to that paper for further details. The stratospheric data assimilation model (STRATAN), that has been developed at NASA's Goddard Space Flight Center (GSFC), is used to generate the transport winds. Briefly speaking, STRATAN utilizes a global atmospheric general circulation model (GCM) with $4^{\circ}$ (latitude) by 50 (longitude) horizontal resolution and 19 levels in the vertical extending from the ground to about $55 \mathrm{~km}$ in altitude. Conventional meteorological data, LIMS temperature data, and rocketsonde data (where available) are used interactively with the general circulation model in the following manner. The GCM is integrated forward six hours from an initial condition that is derived from analysis of available data at the initial time. The new data are then analyzed using the forecast field as the first guess. Another six hour integration of the GCM is made from this new initial condition, and the cycle of six hour integrations with data insertions continues. Thus, wind and temperature fields are generated consistently with both the governing GCM equations and the data. Further details on STRATAN are given in Rood et al. (1989, 1990), Baker et al. (1987), and Takano et al. (1987).

The STRATAN winds are then used in a transport-chemistry model. A spectral transport scheme, with rhomboidal 30 horizontal resolution, is used in the transport/chemistry model. The ozone continuity equation is solved by process splitting in which the transport algorithm is applied first, with the transported ozone field then acted on by the chemistry operator (see Rood et al., 1991). The ozone production and loss are 15-day averages of the daily-averaged terms evaluated in the GSFC two-dimensional model of Douglass et al. (1989). The initial ozone distribution is from LIMS data above 100 mbar with the values below 100 mbar being taken from the aforementioned two-dimensional model. Further details on this transport-chemistry model are given in Rood et al. (1991). 


\section{3. "Satellite Observations"}

Nimbus 7 was put into a sun-synchronous, nearly circular, orbit, with a noon equator crossing on the ascending (that part of the orbit during which the satellite moves toward the North Pole, generally corresponding to daylight conditions) node, and a period of 103.98 minutes. The initial apogee and perigee altitudes were 953 and $940.5 \mathrm{~km}$, respectively. These values changed a bit with time but remained quite close to these values during the life of the LIMS instrument. The LIMS instrument on Nimbus 7 is pointed $146.5^{\circ}$ clockwise from the velocity vector, when viewed from above. For the purpose of simulating LIMS observations, we chose the following parameters: satellite period $=102.8$ minutes; plane angle between the satellite's orbital plane and the earth's equatorial plane $=99.3{ }^{\circ}$; spherical earth's radius $=6,300 \mathrm{~km}$; satellite altitude $=912.8 \mathrm{~km}$; LIMS view angle with spacecraft velocity vector (measured counterclockwise from the spacecraft velocity vector) $=213.50$; period of earth's rotation $=24$ hours. With these values, it is found that exactly fourteen orbits take place in one day, so the satellite's orbit is exactly sun-synchronous.

We "fly" the "satellite " through the evolving model 30 mbar $\mathrm{O}_{3}$ field. In actuality, The $\mathrm{O}_{3}$ fields are saved only every six hours so that at each "satellite observation point," we perform a linear spatial interpolation using the four surrounding $\mathrm{O}_{3}$ grid values and then linearly interpolate to the time of the observation. (We have checked this procedure by repeating the "satellite observation" and mapping calculations with a special data set of shorter duration in which the $\mathrm{O}_{3}$ values were saved every two hours. The differences between the two were seen to be negligible for our purposes.)

For illustration purposes, Figure (1) shows the "ground-track" of the simulated "satellite" for one day, and Figure (2) shows the locus of the simulated "LIMS observation points" for one day.

With these procedures, we generated a mathematically simulated set of "Nimbus 7 LIMS observations" of 30 mbar $\mathrm{O}_{3}$ from the model output. 


\section{Mapping}

The archived LIMS data are generally available in two forms. The first is LIMS Inverted Profile Archival Tapes (referred to as the LAIPAT, and the second is LIMS Map Archival Tape (referred to as the LAMAT). The procedure for generating the LAMAT from the LAIPAT has been described by Haggard et al. (1986). Briefly, speaking, the procedure is as follows. The Kalman filter method (Rodgers, 1976; Kohri, 1981) is used as a sequential estimator to derive time-varying zonal Fourier coefficients (sine and cosine basis functions) at each latitude and altitude. In applying the Kalman filter, some initial information on data variances and the time evolution of the errors of the resulting Fourier coefficients must be specified. For the LIMS data, Haggard et al. (1986) calculated the data variances as a function of latitude and altitude for each 30-day period. The relaxation times were also calculated for each 30-day period for each wave number $(0-6)$ as a function of altitude. These parameters were then used to generate the Fourier coefficients for each 30-day period, and, after 30-day updates of the parameters of the Kalman filter, the Fourier coefficients were generated for the entire LIMS period for all of the measured parameters. These Fourier coefficients can then be used to generate synoptic maps for any desired time.

We have used somewhat simpler methods to generate the maps of the "satellite observations" of the modeled 30 mbar $\mathrm{O}_{3}$. In his analysis of LRIR data, Kohri (1981) determined the variances of wave numbers $0-6$ by their values at $10 \mathrm{mbar}$, the highest level that he could obtain from conventional data. By auto-correlation analysis at $10 \mathrm{mbar}$, he determined that the time constant, $\tau$, was approximately 4 days for wave numbers $0-3$, which dominate at stratospheric levels, and that the use of separate values of $\tau$ for each wave number was not warranted. In our case, we have calculated the variances from the first ten days of our modeled period, January 1, 1979 - January 10, 1979 . We have adopted a wave numberindependent time constant of 3.7 days that we have used in our Kalman mapping.

Thus, when we refer to Kalman-mapped results in this paper, we refer to this Kalman filter mapping procedure being applied to the "satellite observations" of the modeled 30 mbar $\mathrm{O}_{3}$ fields. 


\section{Results}

We want to examine the spatial and temporal variability of the model fields (hereafter referred to as the "model" fields), the mapped "satellite" observations of the model fields (hereafter referred to as the Kalman fields), and the mapped LIMS observations themselves. We have found that the most effective manner for this comparison, is to look at computer video animations of these results. This is, however, impractical for journal presentation, so instead we will be comparing individual mapped fields with one another to compare the spatial structural content of the model results, the Kalman fields, and the LIMS maps. To look at the temporal structure of these results, we will be comparing time series of 30 mbar $\mathrm{O}_{3}$ at selected points.

The results shown here are from a transport-chemistry run that was initialized with the $\mathrm{O}_{3}$ observations on January 1, 1979. The run ended on February 28, 1979. During this period, there occurred a wave 1 minor warming in late January and a major warming in late February. These warmings have been the subjects of extensive study and are probably the most studied of all stratospheric warmings.

Figure (3) shows comparisons between modeled 30 mbar $\mathrm{O}_{3}$ and LIMS observed $30 \mathrm{mbar} \mathrm{O}_{3}$ at $12 \mathrm{Z}$ on January 27,1979 , a day near the peak of the wave number one minor warming while Figure (4) shows the same comparison for February 22, 1979, a day near the peak of the wave number 2 major warming.

Figure (3a) shows the true synoptic map of the model results for $12 Z$ on January 27,1979 , while Figure (3b) shows the "synoptic map" that was constructed by Kalman filtering of the LIMS observations. Only the Northern Hemisphere is shown in these figures since the LIMS results show only weak features in the Southern Hemisphere, and the modeling results are poor there, probably due to the lack of sufficient data to constrain the model. Both the model and LIMS results show a large region of high $\mathrm{O}_{3}$ mixing ratios centered at about $60 \mathrm{oN}$ and $180 \mathrm{oE}$ and a broad region of low $\mathrm{O}_{3}$ mixing ratios centered at about $60 \mathrm{oN}$ and $20{ }^{\circ} \mathrm{E}$. These features show a definite northeast to southwest slope. Much more spatial structure is seen in the modeled $\mathrm{O}_{3}$ field.

In the processing of the LIMS data, only wave number 0 - 6 are retained since, given the number of equatorial crossings of LIMS 
on Nimbus 7, only these low wave numbers can be resolved. We have filtered the model results of Figure (3a) by Fourier decomposing this field and recomposing the wave number $0-6$ components of this field. The resulting Figure (3c), while being noticeably smoother than Figure (3a), still is seen to have much more spatial structure than does Figure (3b), the LIMS data. Finally, Figure (3d) shows the Kalman results for this day. The qualitative agreement between the LIMS observations and the Kalman results at middle and high latitudes is quite good with the wave number one in $\mathrm{O}_{3}$ mixing ratio being reproduced with approximately the same amplitude and placement. The Kalman filter results show weaker spatial gradients though. This is not particularly surprising when one remembers that the spacing between model "observations" was much greater than was the spacing between LIMS observations. The agreement in the tropics is less satisfactory, however, with less structure being observed in the LIMS field than in the Kalman filtered model results. The poorer model results in the tropics will be discussed a bit more later on.

Figures (4a) - (4d) show results for $12 Z$ on February 22, 1979. A very different configuration is seen in Figure (4) than was seen in Figure (3). Figure (4) shows two regions of high $\mathrm{O}_{3}$ mixing ratio, one being centered at about $75 \circ \mathrm{oN}$ and $180^{\circ} \mathrm{E}$ and a more diffuse region of high $\mathrm{O}_{3}$ values lying between about $60 \mathrm{oN}$ and $340 \mathrm{oE}$. Two regions of low $\mathrm{O}_{3}$ mixing ratio centered at about $600^{\circ} \mathrm{N}$ and $75 \circ \mathrm{E}$ and $60^{\circ} \mathrm{N}$ and $270^{\circ} \mathrm{E}$ are also seen. Again, much more structure is seen in the model field than in the LIMS field. The wave number 0 - 6 field is smoother than the model field but shows more small-scale features than does the LIMS field. The Kalman field shows good agreement with the LIMS field at middle and high latitudes. The Kalman field shows more structure in the tropics than was observed by LIMS.

Having thus compared the model spatial structure with the LIMS and Kalman field, we now will look at the temporal behavior of these fields We will do this by looking at time series at particular points. Figure (5) shows times series of $\mathrm{O}_{3}$ at 30 mbar at three points, one in the tropics at $8 \mathrm{oN}$ and $176 \mathrm{oE}$, another at middle altitudes at $31 \mathrm{oN}$ and $176 \mathrm{oE}$, and the other at high latitudes at $77 \mathrm{oN}$ and $176 \mathrm{oE}$. Plotted on this figure are the model time series, the LIMS time series, and the Kalman time series. Good agreement is found between the LIMS and both the model and Kalman time series at the the middle and high latitude points with very poor agreement 
seen between these time series at the tropical point. The poorer agreement in the tropics is due to a variety of factors. The data availability is less in the tropics, so there is less data constraint for the model. The balance between the mass and momentum fields is less in the tropics, and diabatic effects are more important there. At all of the latitudes though, it is clear that the model time series contains much more variability at short time scales than do either the LIMS or the Kalman time series. The wave number 0 - 6 time series (not shown) is found to show somewhat less time variability than the model results but more variability than either the LIMS or the Kalman filter results.

\section{Discussion and Summary}

In the previous sections, we have seen the following general result. The process of satellite sampling and subsequent Kalman mapping of model results filters out the short spatial and temporal content of the model fields. This is most certainly also true with respect to satellite sampling of the actual atmosphere. Furthermore, we have seen that there is good agreement between the mapped "satellite observations" of the model results and the mapped LIMS data at middle and high latitudes. Some other examples of this agreement have already been shown by Rood et al. (1991).

One conclusion of this paper is that the most proper way to compare the results of atmospheric models with satellite observations is to compare "satellite sampling" of the model with satellite sampling of the atmosphere. Another conclusion that can be reached is that the actual distribution of 30 mbar $\mathrm{O}_{3}$ that existed at the time of the LIMS measurements probably resembled the results of the Rood et al. (1991) transport-chemistry model more closely than they resembled the distributions shown in the "synoptic" satellite maps. We reach this conclusion by noting that the distribution of a constituent being transported by a fluid flow shows the type of rapidly evolving "tongues" and "filaments" that are seen in a video animation of the modeled $30 \mathrm{mbar} \mathrm{O}_{3}$ rather than the type of gradual evolution from one large-scale pattern to another that one sees in the video animation of the mapped LIMS or the Kalman results. Thus, we believe that the satellite picture is an accurate portrayal of the large-scale 30 mbar $\mathrm{O}_{3}$ field but that the satellite sampling and mapping have obscured the transport physics that formed this large-scale pattern. 
One particularly intriguing situation that we have seen is the following. In viewing animations of the model fields, we see episodes in which high values of $\mathrm{O}_{3}$ are stripped out of the tropics and are transported northward. In animations of both the LIMS data and the "satellite" observed model, the clear connectivity to the tropics is not seen; however, the behavior of the "satellite" observed model and the LIMS results are very similar. This suggests that streams of subtropical air with high $\mathrm{O}_{3}$ are sheared to scales that are are too narrow and rapidly evolving for the satellite to observe. As the tongue of high $\mathrm{O}_{3}$ air reaches regions of divergence at high latitudes, however, the $\mathrm{O}_{3}$ is once again at spatial and temporal scales that the satellite can resolve. Thus, in this type of situation, the satellite sampling and subsequent mapping shows the end result of the transport event but obscures the transport process itself. This situation illustrates a type of failure in budget calculations that plagued Douglass et al. (1985).

The results of this paper depend on several features of this investigation. Firstly, the field that we have chosen to look at in this paper, $30 \mathrm{mbar} \mathrm{O}_{3}$ in the winter hemisphere, is one that has a great deal of structure given the fact that the chemical lifetime of $\mathrm{O}_{3}$ there is in excess of 100 days. This, coupled with the active dynamics that are present, give rise to sharp gradients and much small-scale structure (e. g., Hsu, 1980). As has been mentioned by Salby (1987), there would probably be less structure in regions where the photochemical time scale is shorter. It is also true that satellitederived temperature fields are probably much better representations of stratospheric structure than are satellite-derived constituent fields since the temperature fields are predominantly in the form of slowly moving large scale planetary waves.

The results of this paper also depend somewhat on the specifics of the sampling and mapping that are implied by the orbit of Nimbus 7 and the observing methods of LIMS. Clearly, for instance, a geostationary satellite is capable of observing fast moving smallscale motions in its viewing region. Also, an instrument with horizontal scanning capability can resolve smaller scale features. The construction of a global "synoptic" map from such scanning observations still presents problems, however. An instrument on an orbiting satellite with cross-track scanning capabilities can measure "almost synoptic" swaths through the atmosphere from each orbit around the earth. The spacing between each "almost synoptic" swath is well separated in time, however. 
As Salby (1989) has pointed out, the best way to generate global "synoptic" maps by satellite is to have multiple orbiting platforms so that, in fact, near synoptic sampling of the global atmosphere is achieved. Another more practical solution, however, is through the use of GCM assimilation procedures. Salby (1989) is correct that the GCM-assimilation products are, ".... an amalgam of model and data ...," but we believe that if one carefully checks the consistency of GCM-assimilation products with available data (including both mapped and unmapped data), it provides the most powerful mapping method for satellite data. It also provides one with fields that are consistent with the governing equations that we believe to be correct, so that one can perform diagnostic studies with its output that cannot be done by other means (see Geller et al., 1990, for example).

\section{Acknowledgements}

Both the Stony Brook and GSFC portions of this work have been supported by grants from NASA's Upper Atmosphere Theory program.

\section{References}

Baker W. E., Bloom S. C., Woolen J. S., Nestler N. S., Brin E, Schlatter, T. W., and Brantstator G. W.

Douglass A. R., Rood R. B., and Stolarski R. S

Douglass A. R., Rood R. B., and Stolarski R. S.
1987 Mon. Weather Rev. $115,272$.
1985

1989
J. geophys. Res. 20, 10693.

J. geophys. Res 94, 9862. 
Geller M. A., Rood R. B., and J. A. Kaye

Haggard K. V., Remsberg E. E., Grose W. L., Russell J. M. III, Marshall B. T., and Lingenfelser

Hsu C.-P. F.

Kohri W. J.
1989

A strategy for using general circulation models and satellite data for improving understanding of the stratosphere. paper presented at the International

Symposium on Middle Atmosphere Studies, Dushanbe, USSR, Nov. 12-19.

1986

Description of Data on the Nimbus 7 LIMS Map Archive Tape Temperature and Geopotential Height NASA Technical Paper 2553.

J. atmos. Sci. 37, 2768.

1981 LRIR observations of the structure and propagation of the stationary planetary waves in the northern hemisphere during December 1975 , Cooperative Ph.D. thesis 63, Drexel Univ and NCAR.

1976 Rev. Geophys. and Space Phys 14, 609.

1989 J. atmos. Sci. 46, 687.

Rood R. B., Allen D., Baker W., Lamich D, and J. Kaye

Rood, R. B., Newman P. A., Lait L. R., Lamich D. J., 1990 and Chan K. R.
Rodgers C. D. 
Rood R. B., Douglass A. R,

Kaye, J. A., Geller M. A., Chi Y.,

Allen D. J., Larson E. M., Nash E. R., and Nielsen J. E.

Salby M. L.

Salby M. L.

Salby M. L.

Salby M. L.

Takano, K, Baker W. E., Kalnay E., 1987

Lamich D. J., Rosenfield J. E., and Geller M. A.
1991 J. geophys. Res. 96, 5055.

1982a J. atmos. Sci. 39, 2577.

$1982 \mathrm{~b} \quad J$. atmos. Sci. 39, 2601.

1987 J. geophys. Res. 92, 14781 .

J. Climate 2, 1091.

J. meteor. soc. Japan 67, 83 . 


\section{Figure Captions}

Figure 1. One day's ground track for the simulated "Nimbus 7."

Figure 2. One day's locus of "observation points" for the simulated "LIMS instrument on Nimbus 7."

Figure 3. (3a): Synoptic map of 30 mbar $\mathrm{O}_{3}$ field produced by the transport-chemistry model for $12 Z$ on January 27, 1979. (3b): LIMS map of 30 mbar $\mathrm{O}_{3}$ for $12 \mathrm{Z}$ on January 27 , 1979.

(3b): 30 mbar $\mathrm{O}_{3}$ map corresponding to wave numbers 0 - 6 of model results shown in Fig. (3a).

(3c): "Synoptic" map of 30 mbar $\mathrm{O}_{3}$ at $12 \mathrm{Z}$ on January 27 , 1979 , by Kalman filtering of the simulated "observations of the modeled fields.

Figure 4. Same as Figure (3), but for $12 \mathrm{Z}$ on February 22, 1979.

Figure 5. Time series for 30 mbar $\mathrm{O}_{3}$ for days 10 - 56 (January 10 - February 25, 1979) where the long dashed lines show the model results; the dotted lines show the LIMS results; and the solid lines show the Kalman filtered results of the simulated "observation" of the model results. (5a) is for $8 \mathrm{oN}, 176{ }^{\circ} \mathrm{E}$. (5b) is for 31 oN, 176 oE. (5c) is for 77 o N, 176 oE. 


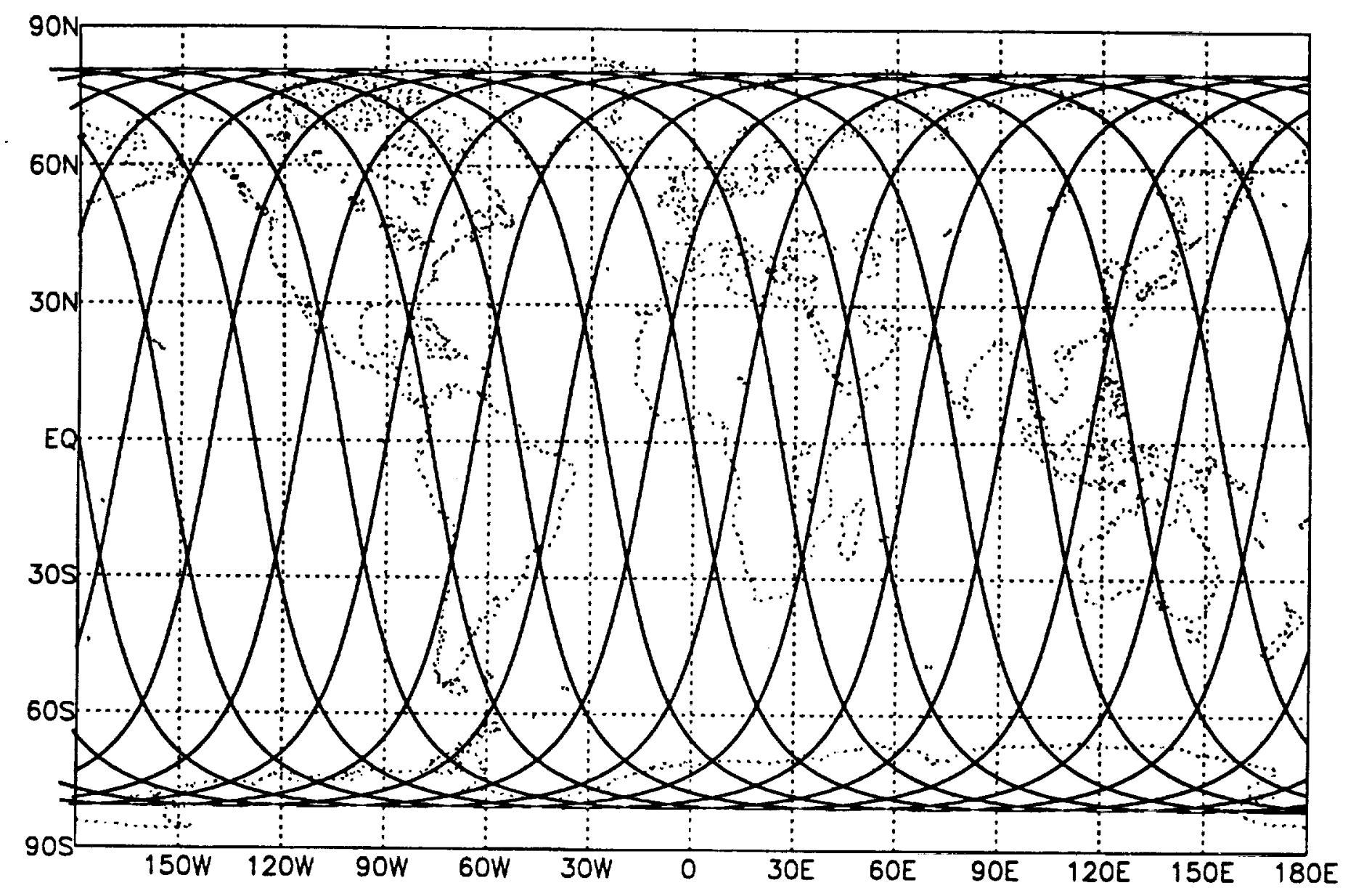

Figure 1 


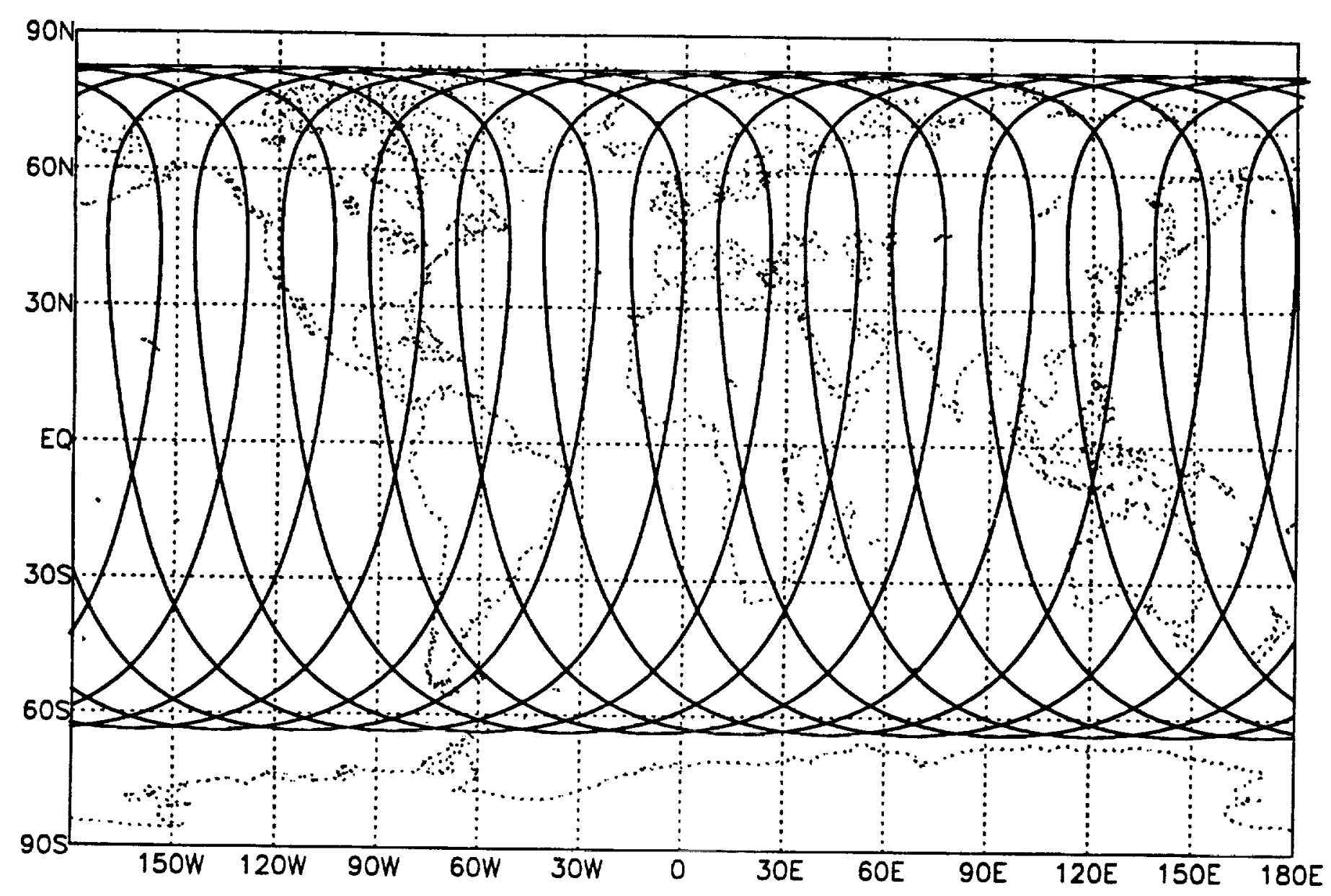

Figure 2 

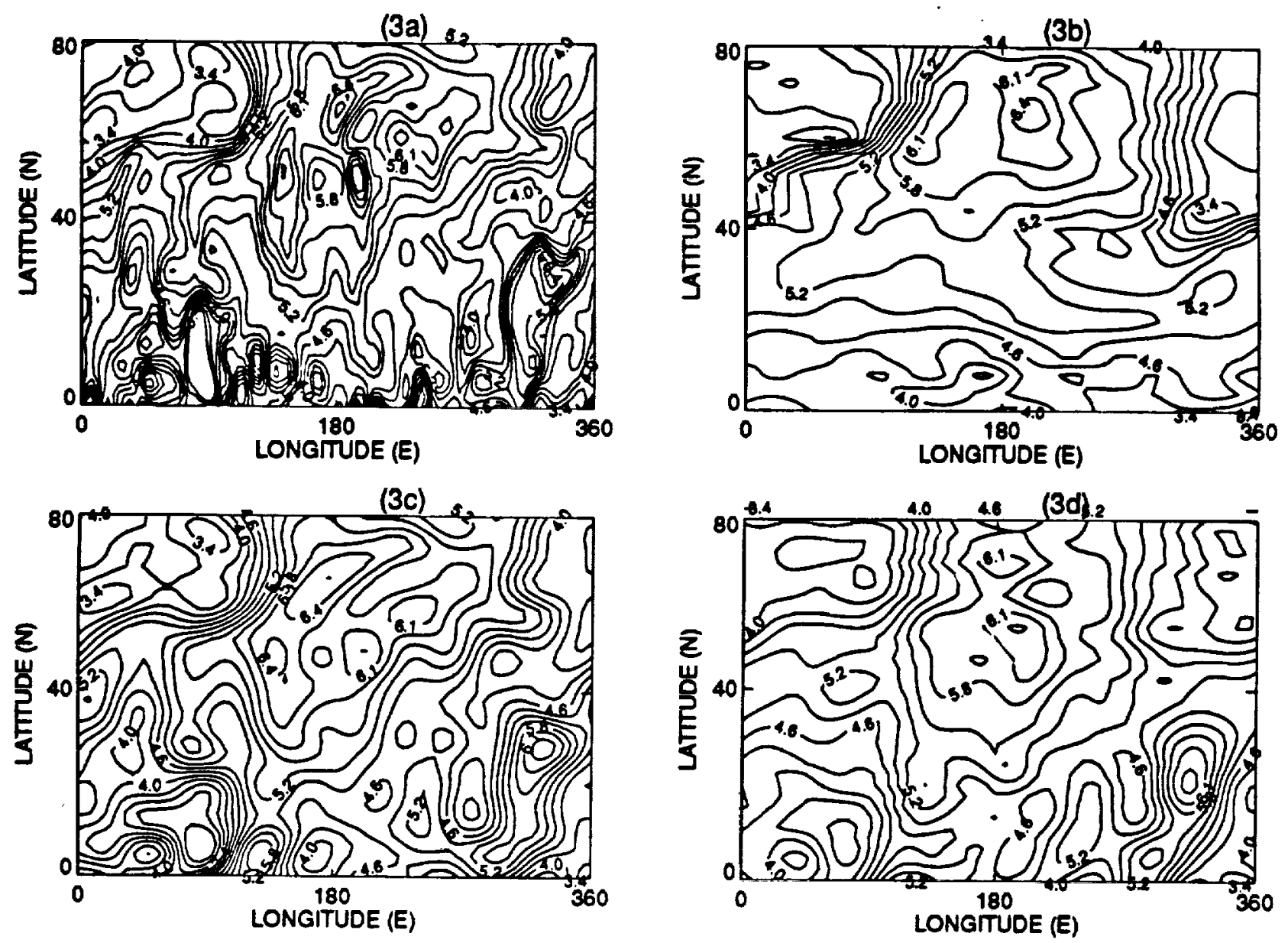

Figuie 3 

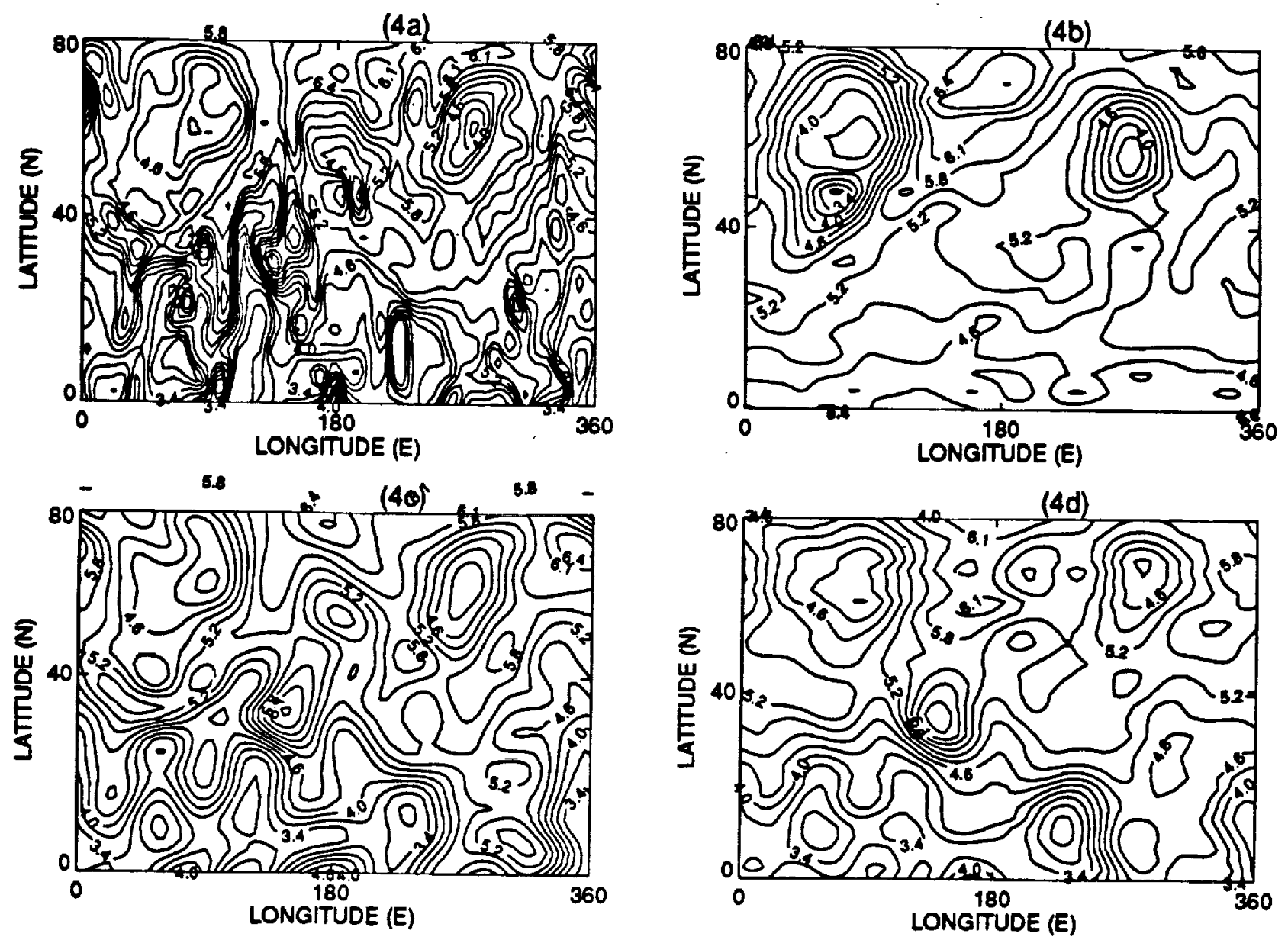

Figure 4 
(5a)

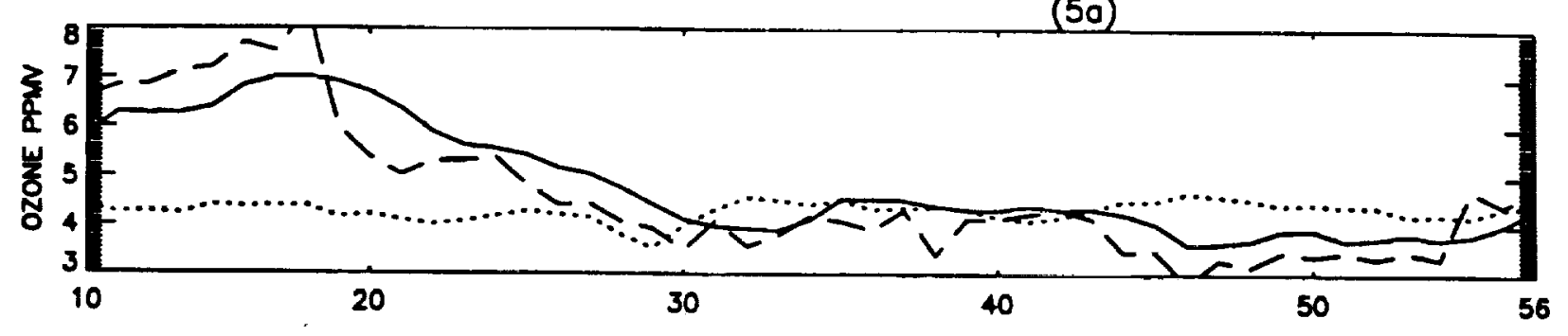

(5b)

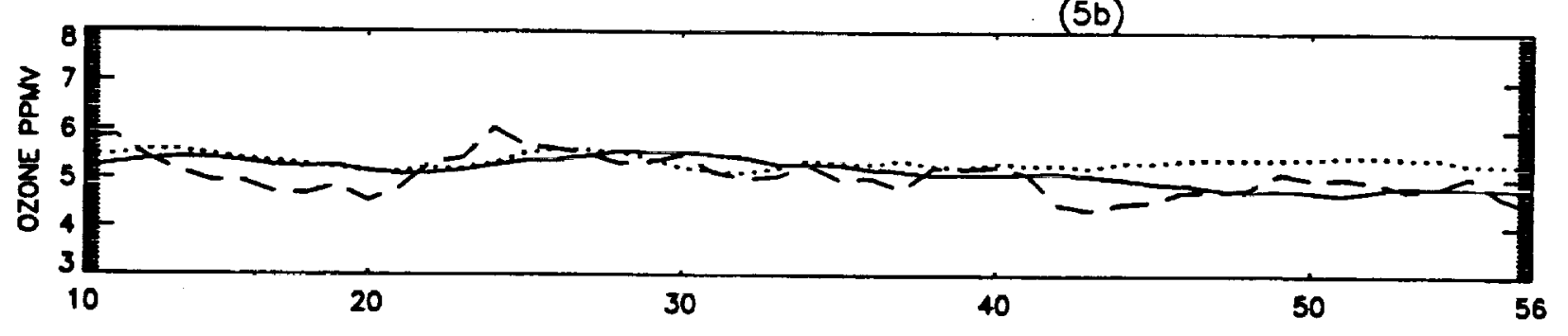

(5c)

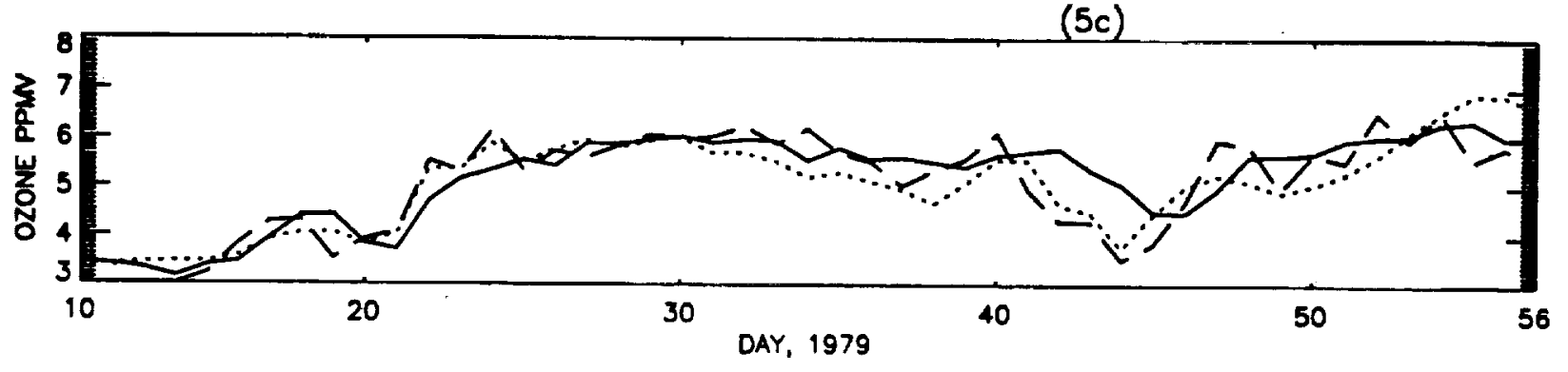

Figure 5 